\title{
Visualization of 3D Hydrogeological Data in the Web
}

\author{
Alexander $\mathrm{KMOCH}^{1}$ and Hermann $\mathrm{KLUG}^{2}$ \\ ${ }^{1}$ AUT University, Auckland / New Zealand · alexander.kmoch@aut.ac.nz \\ ${ }^{2}$ Interfaculty Department of Geoinformatics - Z_GIS, University of Salzburg / Austria
}

This contribution was double-blind reviewed as full paper.

\begin{abstract}
New Zealand's groundwater resources are inadequately understood to effectively support an integrated and sustainable freshwater management. To appropriately characterise the groundwater aquifers, a lot of environmental data are required; among them (hydroggeological datasets. Usually, proprietary software products are used to establish, visualise and analyse the geological underground. This prevents a broad and public distribution of information to those who need it. In this manuscript we demonstrate a framework to enable a web-based (platform independent) retrieval and visualisation of three-dimensional information via the web browser. We link distributed data and processing services to prepare an on-demand 3D visualisation of geological and hydrological data.
\end{abstract}

\section{Introduction}

Freshwater management is an important task for the regional councils in New Zealand, which is legislated by the Resource Management Act (MINISTRY FOR THE ENVIRONMENT NEW ZEALAND 2013). In the last decade, regional councils face an increasing demand on freshwater resources from agriculture. While most surface waters are already allocated, the actual volumes of available groundwater resources are not well understood (WHITE \& REEVES 2002; LOWRY et al. 2003). The authors develop a web-based framework for discovery, access, visualisation and analysis of hydrogeological data to support the characterisation of New Zealand's aquifers. This framework provides functionality for discovery and data access and is implemented and demonstrated within the SMART groundwater portal ${ }^{1}$ (KLUG \& KMOCH accepted 2014). A web mapping example is shown in Fig. 1. As foundational principles, the standardisation efforts of the Open Geospatial Consortium (OGC 2014) and World Wide Web Consortium (W3C 2014) are leveraged to make hydrogeological data accessible, as well as to lift the analysis and visualization processes into the web and to make it independent of vendor- and platform-specific data access, processing, and visualization boundaries.

\footnotetext{
${ }^{1}$ SMART groundwater portal: http://portal.smart-project.info
} 
Typical web map viewing applications display 2D representations of geospatial groundwater data layers via OGC compliant Web Map Service (WMS), Web Feature Service (WFS) and Web Coverage Service (WCS) layers from remote servers (Fig. 1) and graphs for hydrological time-series data from Sensor Observation Services (SOS) (BRODARIC et al. 2011; KMOCH et al. 2012). Typically data from SOS servers are represented and grouped by their sampling locations, i.e. sensor stations. Locations are printed on a web map and the time-series data for available phenomena are then requested and graphed (BRÖRING et al. 2011). To visualise the spatio-temporal distribution of phenomena, geostatistical algorithms use near-real-time SOS data and are exposed via OGC Web Processing Services (WPS) and thus can be integrated into web based visualisation systems (PEBESMA et al. 2011; CASTRONOVA et al. 2013a). WPS allows distributed and chained processing in the web, and computing power can be extended into the cloud, i.e. multiple instances run in parallel on different servers in the internet. This concept has also been addressed as Service Oriented Architecture (GIULIANI et al. 2012; CASTRONOVA et al. 2013b) to connect web based spatial data provisioning and web processing services.

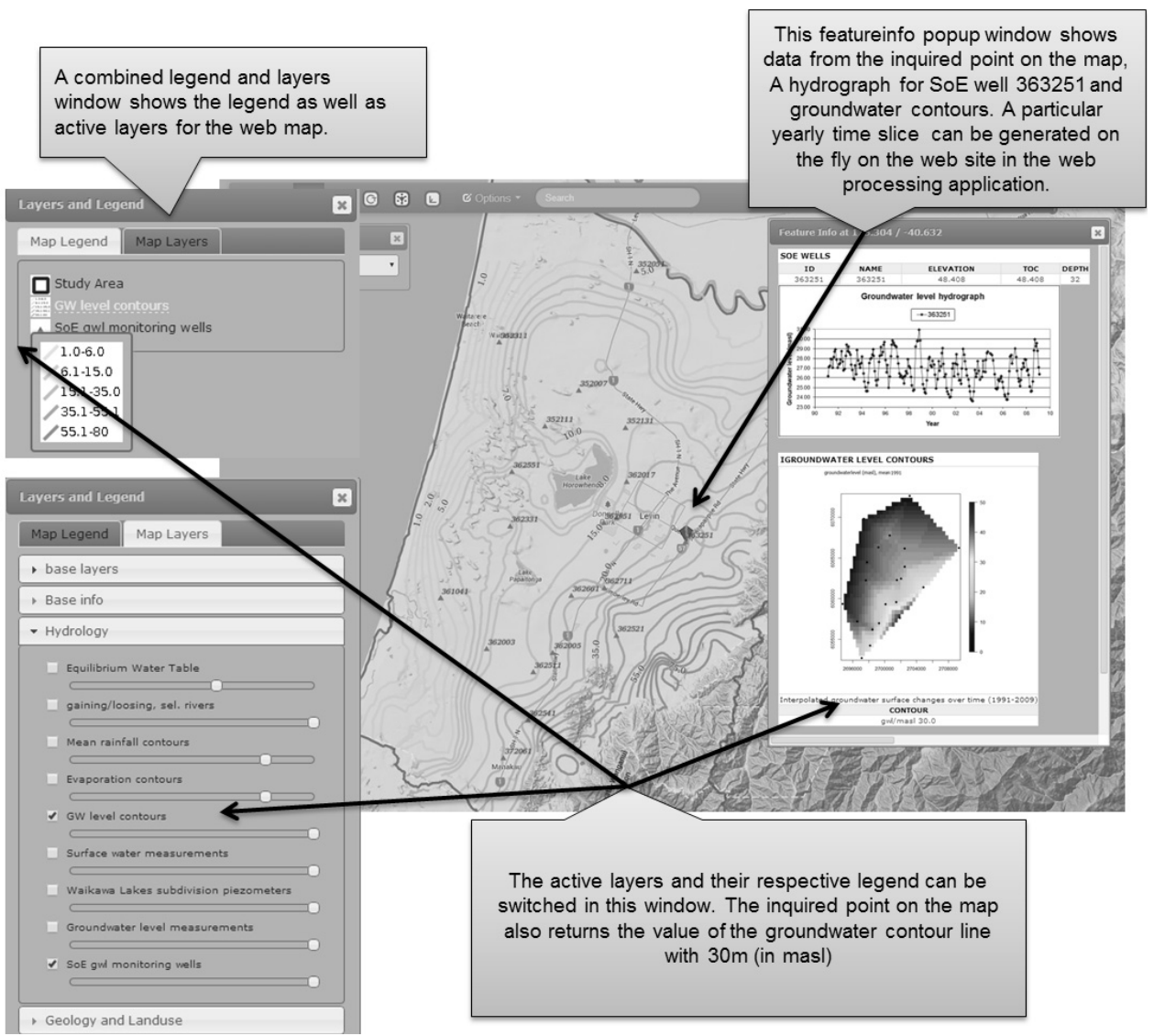

Fig. 1: Web map view of the Horowhenua case study area, New Zealand, groundwater time-series and contours, SMART groundwater portal (KLUG \& KMOCH accepted 2014) 
To keep track of the state of the actual visible map with its active layers, zoom, bounding box and styling properties, as well as to deliver predefined collated maps, Web Map Context WMC (OGC 2005) documents are used to persist the current map state configuration. WMC documents provide a structured and standard-compliant way to define a map context, holding references and styling declarations for spatial data sources. Furthermore it is also possible to reference other geospatial web services, like Web Coverage Service (WCS) or Web Map Tile Service (OGC 2010), which provide 2D map representation capabilities. However, the integration of a time series data source (e.g. SOS) with WMC has not been demonstrated yet.

For the web-based visualisation of 3D geospatial data and models, several technologies, standards and methodologies have been described in the literature lately (STOTER et al. 2011; GRÖGER \& PLÜMER 2012; SCHMIDT \& MAY 2012; COX et al. 2013; EVANS et al. 2014). X3D (ISO/IEC 2008) is the successor of the ISO standard VRML97, and has been demonstrated in the web browser, particularly with x3dom (X3DOM 2014). Alternative developments that show 3D geological, hydro-climate, or hydrogeological maps in the web make use of native WebGL (COX et al. 2013), or the OGC 3D draft standards Web Perspective View Service and Web 3D service (SCHMIDT \& MAY 2012). While all these approaches render static spatially explicit datasets into a $3 \mathrm{D}$ scene, none considered a dynamic integration with on-the-fly generated geostatistical datasets based on time-series data.

The case study area is the Horowhenua municipal district, located on the west coast of the lower North Island and part of the Manawatu-Wanganui Region (Horizons). Lake Horowhenua is the defining natural feature in this rather flat landscape, which becomes slightly hillier towards the south east. A 3D geological model was created as part of a groundwater budget assessment for the Horizons Regional Council (WHITE et al. 2010a; WHITE et al. 2010b). The refined datasets for this area include groundwater level timeseries from monitoring wells (via SOS), and four geological layers (Greywacke, Holocene, and quaternary layers Q6 and Q2Q3Q4). In this paper we describe how the time-series and geological layer datasets are made accessible through standards compliant web services, and integrated into $3 \mathrm{D}$ data visualisation in the web.

\section{Material and Methods}

\subsection{Using SOS services in Web Map Context documents}

In SOS 2.0 terminology, an observation offering groups collections of observations (i.e. the time-series data), and prescribes that each observation offering in SOS can only have a 1:1 cardinality to a so called procedure. Following OGC SOS and Observations and Measurements (O\&M) semantics, a procedure can be a sensor, i.e. a system, or a process or algorithm that describes how the observation was made. In the web map context (WMC) conceptual model, each referenced resource is described as a layer. While SOS does not know the concept of a layer, we use the observation offering equivalently instead. Thus, we group all observations for each single phenomenon as one offering. This is necessary to represent the spatial distribution of one observed property via one offering as opposed to a grouping by sensor stations. Therefore each procedure is described as a process of data 
generation for all measurement stations for the same observed property. Both the " $<$ Extension $>$ " as well as the " $<$ DimensionList $>$ " elements from the WMC specification allow the possibility to also describe temporal boundaries for the time-series data. This can be done via the OGC compliant gml:TimeInstant, or gml:TimePeriod elements.

The 3D geological model layers are only available as plain text grid files and are represented as their top boundaries. Therefore they represent spatial coverages with height as their attribute values. This allows publishing such data sets as standardised OGC WCS coverage layer with software like GeoServer².

\subsection{On-demand 3D scene generation}

We describe a three step process from a $2 \mathrm{D}$ web mapping context to a $3 \mathrm{D}$ view with a dynamic on-demand creation of a X3D scene graph document, based on spatio-temporal data services described in an OGC compliant WMC document.

The 2D web mapping application holds the actual context of the visible layers and sampling locations of the case study area. The known 4-layer geological underground model is only visualised as an area in the $2 \mathrm{D}$ view. The SOS layer is based on an observation offering, e.g. the groundwater levels for the Horowhenua area. The top layer of the 3D geological model represents the actual land surface.

Fig. 2 represents a schematic view of the method. The WMC document is submitted to a managing framework process (step \#1), which parses the layer definition. This framework process controls the overall $3 \mathrm{D}$ scene creation. If a SOS layer definition is found in the WMC document, it dispatches a WPS request including the SOS service URL, observation offering, and time dimension, and expects a WCS layer reference as a return value from the dispatched, distributed WPS algorithm in step \#2.

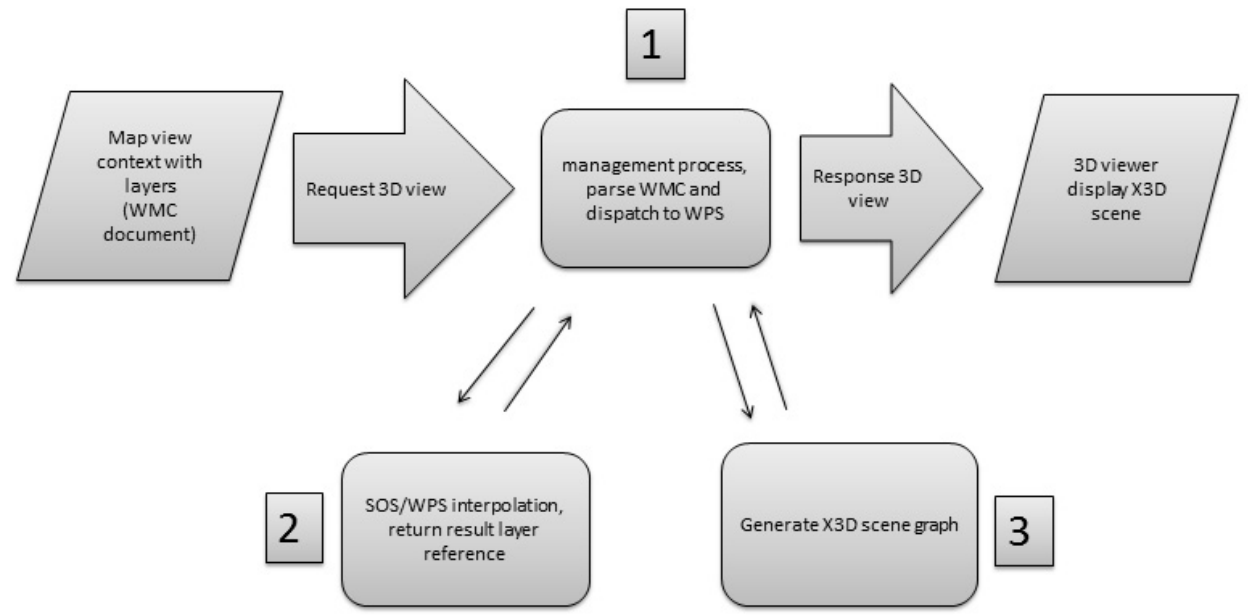

Fig. 2: Process of scene generation

2 GeoServer website: http://geoserver.org 
This WPS algorithm constructs and issues a SOS_GetObservation request, retrieves the response data, and applies a linear spatial interpolation technique to create a spatially continuous coverage dataset. This dataset is made accessible via WCS, and the reference to the WCS layer resource is returned to the framework process.

In step \#3 the framework process requests an X3D object for each layer from another WPS algorithm and assembles the final X3D scene graph based on the definitions in the WMC document.

The fully constructed X3D scene graph is returned to a viewer application. This switches to a 3D mode and loads the 3D scene via $\mathrm{x} 3 \mathrm{dom}$ into the web browser, which then can be explored by the user.

\section{Results}

We described a methodology of on-demand 3D visualisation of hydrogeological data in the web. A framework process manages the interaction between data and processing services. WMC has successfully been trialled and tested to demonstrate the integration of spatiotemporal observation data via SOS into a web mapping context. The initial message to the framework process is the request for a $3 \mathrm{D}$ view of a map context, which is defined via a Web Map Context (WMC) document. Source datasets are delivered via different OGC compliant web services. In Fig. 3 a SOS layer is described in a WMC document that would map observations from the offering "groundwater_levels".

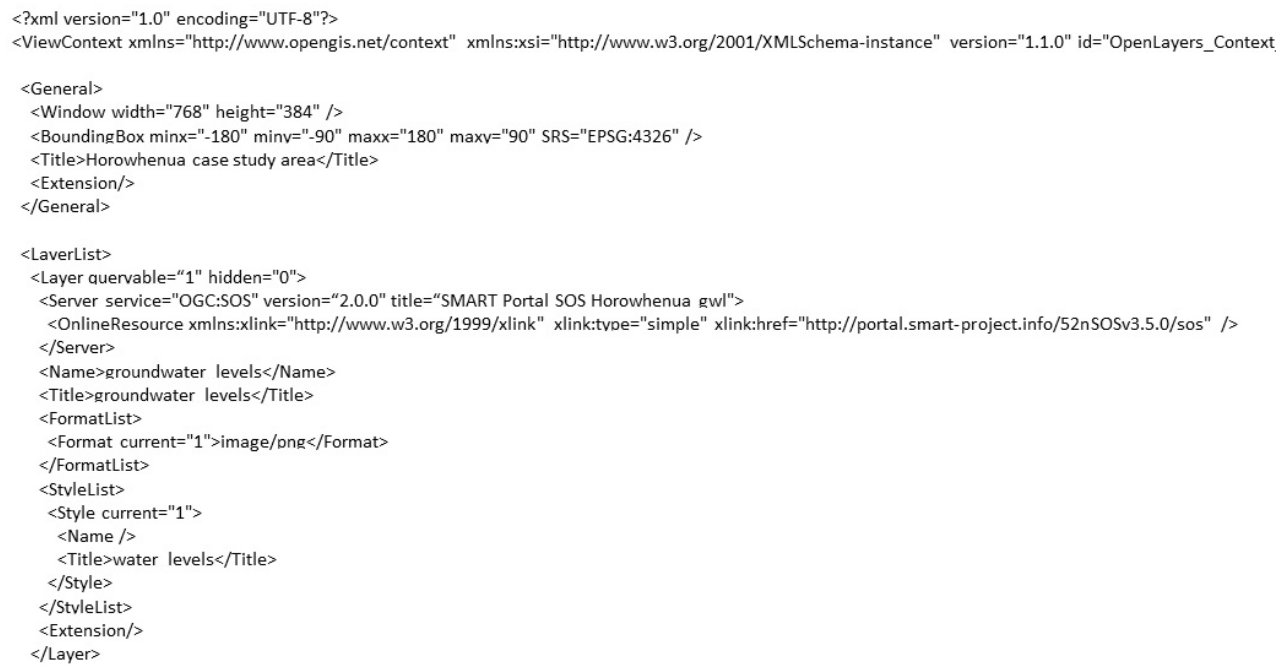

Fig. 3: XML example of WMC document with experimental SOS notation

Furthermore, the generation of X3D elements and the assembly of a full X3D scene graph based on OGC data sources via WPS algorithms has been described. Table 1 demonstrates how the OGC service and data elements are mapped and transformed to $\mathrm{X} 3 \mathrm{D}$ scene 
elements. Here, the WMC document was also re-used to define the data sources, which were subsequently inquired and the returned with the data transformed into the respective $\mathrm{X} 3 \mathrm{D}$ elements.

Tab. 1: Mapping of data type to visual type

\begin{tabular}{|l|l|}
\hline OGC data source & X3D element \\
\hline WMS layer & Image draping nodes for (top) surface \\
\hline $\begin{array}{l}\text { 3D geological model layer, } \\
\text { WCS elevation dataset }\end{array}$ & ElevationGrid as an evenly spaced grid \\
\hline SOS layer, groundwater level & The interpolated WCS layer will be treated as elevation \\
\hline $\begin{array}{l}\text { SOS layer, other physical or } \\
\text { chemical property }\end{array}$ & The interpolated WCS layer will be treated WMS \\
\hline
\end{tabular}

Fig. 4 shows three 3D scenes, which are generated from the same web map context including the different geological layers, for example in a) the New Zealand topographical map and the interpolated groundwater level surface. In all three different configurations the geological layer models are extruded by the factor 30 to make their surface characteristics more visible. In Fig. 4, example b) all elements are evenly exploded by factor 200 with the groundwater surface and the topographical map atop for better visibility. In examples a) and c) the greywacke layer second from the top represents the geological basement and is drawn separately. The other geological layers are placed above but keep their vertical reference to each other. In example c) the interpolated groundwater surface is then also placed vertically correct within the geological layers. The topographical map is placed below to provide the geographic location context for the 3D scenes.
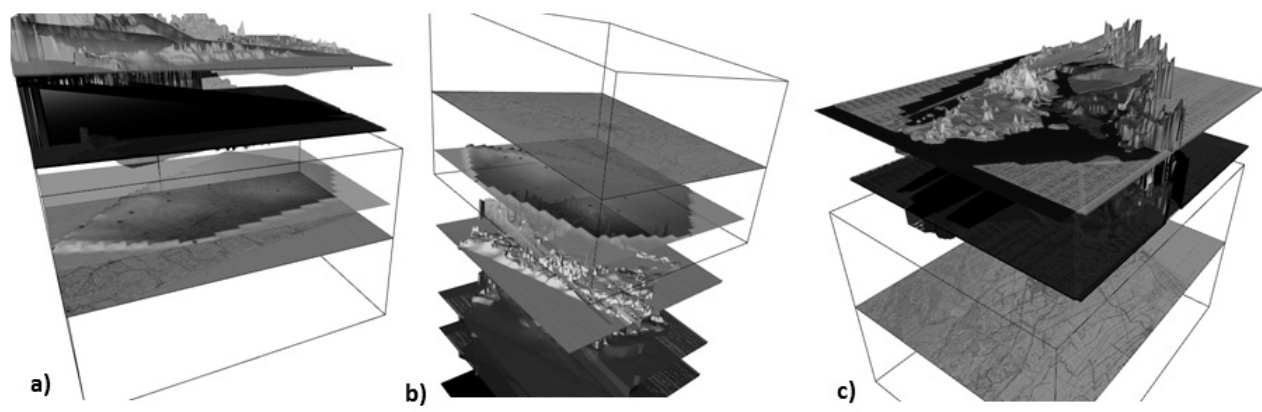

Fig. 4: Three configurations for 3D scene generation from a provided map context, including extruded and exploded geological layers, the New Zealand topographical map and the interpolated groundwater level surface 


\section{Discussion and Conclusion}

Three-dimensional web visualisation capabilities for environmental data in New Zealand are presently limited, and established 3D models are based on cost-intensive proprietary desktop software solutions. With this 3D visualisation method, based on distributed data sources and scene creation framework services, we enable insight into the subsurface, and thus a better understanding of available (ground) water resources. This will support policy making, as well as provide a common ground for competitive groundwater management and use. The integration of monitoring observation time series will characterise input and output relations of the catchment in near real-time.

The exercise of declaring a SOS layer based on observation offering in a WMC context document has not been discussed in the literature, although the specification explicitly states the possibility to do so. However, regarding the flexibility of the specifications under the SWE framework - especially SOS, O\&M, and Sensor Model Language (SensorML) this concept can only represent one of many possible and valid configurations. Yet, this contribution can be proposed to the community to discuss a broader range of applicable use cases.

The implicit spatial property interpolation from the spatio-temporal sampling data is a special application, and an experimental way of visualising time-series in a web based distributed Spatial Data Infrastructure. The same applies to specific definition in the WMC context document, based on the observation offering of the SOS service, which is then forwarded to a WPS instance. This implies a prior knowledge in a loosely-coupled Service Oriented Architecture (SOA), which only relies on the interface descriptions at runtime. However, the manner of chaining the distributed WPS, to generate the interpolated datasets, as well as the $\mathrm{X} 3 \mathrm{D}$ elements and the scene graph, resemble an architecture that subsequently aggregates the results of the distributed, yet integrated services.

\section{Outlook}

The initial assumption of how a SOS has to offer its data and the mapping of data sources into $3 \mathrm{D}$ space is too narrow to apply to an open data services federation, particularly if it is not known after which conceptual model the data is provided. Here, the recent developments of the semantic web offer methodologies to describe the conceptual model and semantic meaning of the data. Therefore such semantics can significantly enrich the discovery and mapping of data to processes. Thereby, a necessity of vocabulary services and more advanced semantics arise, to support such chained distributed processes.

Prior knowledge of the type and characteristics of the observed phenomena can also improve interpolation methods. Groundwater level interpolation with Indicator Kriging methods could for example use elevation data and geological layer models as external drift variables. Based on the integration of further hydro-climate time-series like water abstraction, precipitation, and evapotranspiration data, the web enablement of more specialised models and algorithms can increase, or even just enable predictions of changes in water levels, and lead to a more sustainable and better informed management of water resources. 


\section{Acknowledgements}

The authors wish to acknowledge funding for the SMART project from the Ministry of Business, Innovation, and Employment (MBIE), New Zealand. We are also very thankful to the stakeholders participating in this project and providing valuable input for the tool developments. We acknowledge our colleagues for providing us with data for inclusion in the SMART groundwater portal. Use of the Horowhenua data in the SMART groundwater portal has been courtesy of the Horizons Regional Council and we thank them for their assistance.

\section{References}

Brodaric, B., Sharpe, D., Boisvert, E., Logan, C., Russell, H., Julien, H., SmirNOFFM, A. \& LÉTOURnEAU, F. (2011), Groundwater Information Network: Recent developments and future directions. Geohydro 2011. Joint Meeting of the Canadian Quaternary Association and the Canadian Chapter of the International Association of Hydrogeologists. Quebec City, Quebec.

Bröring, A., Echterhoff, J., Jirka, S., Simonis, I., Everding, T., Stasch, C., Liang, S. \& Lemmens, R. (2011), New Generation Sensor Web Enablement. Sensors, 11 (3), 2652-2699.

Castronova, A. M., Goodall, J. L. \& Elag, M. M. (2013a), Models as web services using the Open Geospatial Consortium (OGC) Web Processing Service (WPS) standard. Journal of Environmental Modelling \& Software, 41, 72-83. http://dx.doi.org/10.1016/j.envsoft.2012.11.010.

Castronova, A. M., Goodall, J. L. \& ElaG, M. M. (2013b), Models as web services using the Open Geospatial Consortium (OGC) Web Processing Service (WPS) standard. Journal of Environmental Modelling \& Software, 41, 72-83. http://dx.doi.org/10.1016/j.envsoft.2012.11.010.

Cox, M. E., James, A., Hawke, A. \& Raiber, M. (2013), Groundwater Visualisation System (GVS): A software framework for integrated display and interrogation of conceptual hydrogeological models, data and time-series animation. Journal of Hydrology, 491, 56-72. http://dx.doi.org/10.1016/j.jhydrol.2013.03.023.

Evans, A., Romeo, M., Bahrehmand, A., Agenjo, J. \& Blat, J. (2014), 3D graphics on the web: A survey. Computers \& Graphics, 41, 43-61. http://dx.doi.org/10.1016/j.cag.2014.02.002.

GiUliani, G., NAtivi, S., LehmanN, A. \& RAY, N. (2012), WPS mediation: An approach to process geospatial data on different computing backends. Computers \& Geosciences, 47, 20-33. http://dx.doi.org/10.1016/j.cageo.2011.10.009.

GRÖGER, G. \& PLÜMER, L. (2012), CityGML - Interoperable semantic 3D city models. ISPRS Journal of Photogrammetry and Remote Sensing, 71, 12-33. http://dx.doi.org/10.1016/j.isprsjprs.2012.04.004.

ISO/IEC (2008), Extensible 3D (X3D) - ISO/IEC 19775-1.2:2008. X3D. http://www.web3d.org/x3d/specifications/, ISO/IEC.

KLUG, H. \& KMOCH, A. (ACCEPTED 2014), A SMART Groundwater Portal: A user centred knowledge inventory for information search, discovery, access and visualisation. Journal of Hydrology (NZ). 
KMOch, A., KLug, H. \& White, P. (2012), FreshwateR resources management: first steps towards the characterisation of New Zealand's aquifers. In: JEKEL, T., CAR, A., STROBL, J. \& GRIESEBNER, G. (Eds.), GI_Forum 2012: Geovizualisation, Society and Learning. Berlin/Offenbach, Wichmann, 37̄6-385.

Lowry, T. S., Bright, J. C., Close, M. E., Robb, C. A., White, P. A. \& Cameronm S. G. (2003), Management gaps analysis: a case study of groundwater resource management in New Zealand. International Journal of Water Resources Development, 19 (4), 579592. 10.1080/0790062032000161382.

MinisTRY FOR THE ENVIRONMENT NEW ZEALAND (2013), Resource Management Act 1991. 1991 No 69. Zealand, M. f. t. E. N. Wellington, New Zealand, Ministry for the Environment New Zealand, 1991, 69, p. 805.

OGC (2005), OGC Web Map Context, WMC 1.1.0. http://www.opengeospatial.org/standards/is; The Open Geospatial Consortium (OGC).

OGC (2010), OGC Web Map Tile Service Implementation Standard, WMTS 1.0.0. http://www.opengeospatial.org/standards/is; The Open Geospatial Consortium (OGC).

OGC (2014), OGC Standards. http://www.opengeospatial.org/standards; Open Geospatial Consortium.

Pebesma, E., Cornford, D., Dubois, G., Heuvelink, G. B. M., Hristopulos, D., Pilz, J., STÖHLKER, U.; Morin, G. \& SKøIEN, J. O. (2011), INTAMAP: The design and implementation of an interoperable automated interpolation web service. Computers \& Geosciences, 37 (3), 343-352. 10.1016/j.cageo.2010.03.019.

SCHMIDT, B. \& MAY, M. (2012), TRITURUS: A Java-based Framework for 3D GeoVisualization Applications. White Paper.

Stoter, J., Vosselman, G., Goos, J., Zlatanova, S., Verbree, E., Klooster, R. \& Reuvers, M. (2011), Towards a National 3D Spatial Data Infrastructure: Case of The Netherlands. Photogrammetrie - Fernerkundung - Geoinformation, 6/2011, 405-420. $10.1127 / 1432-8364 / 2011 / 0094$.

W3C (2014), The World Wide Web Consortium (W3C). http://www.w3.org/; The World Wide Web Consortium.

White, P. A. \& ReEves, R. R. (2002), The volume of groundwater in New Zealand 1994 to 2001. GNS Science Client Report 2002/79. Taupo, New Zealand.

White, P. A., Raiber, M., Della Pasqua, F., Zarour, H. \& Meilhac, C. (2010a), Threedimensional geological model of the Horowhenua area. GNS Science Consultancy Report 2009/310. Taupo, New Zealand.

White, P. A., Raiber, M., Zarour, H., Meilhac, C. \& Green, S. (2010b), Horowhenua water resources: water budget and groundwater-surface WATER INTERACTION, GNS Science Consultancy Report 2010/22.

X3DOM (2014), X3DOM - an experimental open source framework and runtime to integrate HTML5 and declarative 3D content. http://www.x3dom.org/?page_id=2. 